\title{
Riesgo cardiovascular y su relación con el estilo de vida en los estudiantes de la Facultad Ciencias de la Salud de una Universidad Privada Lima, 2017
}

\section{Cardiovascular risk and its relationship with the lifestyle in students of the faculty of health sciences of a private university in Lima, 2017}

\author{
Tania Zhunaula Guamán ${ }^{1}$, Charo Nátali Huzco Rutti²
}

\begin{abstract}
RESUMEN
Objetivo: Determinar el riesgo cardiovascular y su relación con el estilo de vida en estudiantes de la Facultad de Ciencias de la Salud de una Universidad Privada de Lima, 2017. Materiales y métodos: Estudio de tipo cuantitativo, descriptivo, correlacional y transversal. Participaron 183 estudiantes seleccionados por muestreo no probabilístico, se aplicó la encuesta "Estilo de vida saludable" y una ficha de datos antropométricos. Resultados: El $67.3 \%$ son mujeres y el $100 \%$ son solteros. El $85.2 \%$ comprenden una dispersión de edad entre 18 a 23 años y $49.2 \%$ son de la costa. Con respecto a la condición laboral, $77.6 \%$ son dependientes y $50.3 \%$ son de cuarto año. En las dimensiones de estilo de vida se encontró que el $48.6 \%$ mantiene una condición física y deporte poco saludable. El $50.8 \%$ son saludables en recreación y manejo de tiempo libre. En consumo de drogas, alcohol y tabaco el $85.8 \%$ son muy saludables. El $66.1 \%$ en la dimensión de sueño son saludables. El $56.3 \%$ se mantienen saludables en hábitos alimentarios. En autocuidado y cuidado médico $55.7 \%$ son poco saludables. No se evidenció relación estadísticamente significativa entre RCV y el estilo de vida saludable $(p=0.234)$. Los hábitos alimenticios tienen relación estadísticamente significativa con el RCV $(p=0.012)$. Conclusiones: El $82 \%$ de los estudiantes son saludables y $54 \%$, presentó un riesgo cardiovascular moderado. No se encontró correlación estadísticamente significativa $(p=0.234)$ entre RCV y estilo de vida, sin embargo, si se encontró correlación entre RCV y hábitos alimenticios $(p=0.012)$.
\end{abstract}

Palabras claves: Riesgo cardiovascular, estilo de vida, correlación.

\section{ABSTRACT}

Objective: To determine cardiovascular risk and its relationship with the lifestyle of students from the Faculty of Health Sciences of a Private University in Lima, 2017. Materials and methods: It was a quantitative, descriptive, correlational and transversal study, in which 183 students selected by nonprobabilistic sampling participated. There were applied the "Healthy lifestyle" and an anthropometric data sheet. Results: From the sample population, $67.3 \%$ are women and $100 \%$ are single, $85.2 \%$ are aged between 18 and 23 years and $49.2 \%$ are from the coast. According to the labor condition, $77.6 \%$ are dependent and $50.3 \%$ are in fourth year. In the lifestyle dimension, it was found that $48.6 \%$ keep an unhealthy physical and sport condition. Also, $50.8 \%$ are healthy in recreation and free time management. $85.8 \%$ are very healthy in drug, alcohol and tobacco consumption. $66.1 \%$ are healthy in the dream dimension and $56.3 \%$ remain healthy in eating habits. $55.7 \%$ are unhealthy in self-care and medical care. It was not found a statistically significant relationship between CVR and healthy lifestyle $(p=0.234)$. Eating habits have a statistically significant relationship with CVR $(p=0.012)$. Conclussion: We conclude that $82 \%$ of the students are healthy and $54 \%$ presented a moderate cardiovascular risk. It was not found a statistically significant correlation between CVR and lifestyle $(p=0.234)$, nevertheless it was found a correlation between CVR and eating habits $(p=0.012)$.

Keywords: Cardiovascular risk, lifestyle, correlation

${ }^{1}$ Lic. Nutrición, Universidad Peruana Unión, Lima, Perú.

${ }^{2}$ Nutricionista, Empresa ADN SAC, Asesoría en Deporte y Nutrición, Lima, Perú. 


\section{INTRODUCCIÓN}

La Organización Mundial de la Salud (OMS) (1) denominó a las enfermedades cardiovasculares (ECV) como la principal causa de muerte en el mundo. El riesgo cardiovascular es la probabilidad que tiene el individuo de padecer una enfermedad, éste depende de los factores de riesgos que estén presentes. El Factor de riesgo, término utilizado por primera vez por Thomas R. Dawber en 1961 y definido en epidemiología como la situación de una persona de contraer una enfermedad. Factor de riesgo cardiovascular es una característica biológica que incrementa la posibilidad de padecer una enfermedad, concepto dado en el estudio Framingham (predictor de riesgo cardiovascular), hacía más de 5 décadas $(2,3)$.

En el 2012 las ECV fueron causa de 31\% (17.5 millones de personas) de muertes registrados a nivel mundial. Las principales causas son la obesidad, hipertensión, aterosclerosis y diabetes mellitus (4), esta situación se relaciona con los cambios de estilos de vida inadecuados tales como alimentación desbalanceada, falta de actividad física, consumo de sustancias nocivas, pocas horas de descanso, entre otros (5).

La OMS (6), menciona que el estilo de vida relacionado con la salud se basa en la interacción entre las condiciones de vida y los patrones individuales de conducta determinados por factores socioculturales y características personales. El estilo de vida es una variable fundamental que afecta de manera positiva o negativa en la salud según la edad (7) se ha descubierto que la mayoría de hábitos de vida, ya sean saludables o no, se obtienen en edades tempranas (8) y los jóvenes son los que se encuentran en esta etapa, clave para la adopción de estilos de vida (9).los riesgos cardiovasculares se identificaba en personas adultas, sin embargo, en la actualidad la población joven lo padece con frecuencia (10), teniendo en cuenta un factor determinante; la acumulación excesiva de la masa grasa, diagnosticado por las medidas antropométricas según la circunferencia de cintura (11).

Los estilos de vida han producido cambios en los patrones de alimentación (7). Se conoce que existe relación entre los factores de riesgo cardiovasculares (FRCV) y los estilos de vida (5). La mayor parte de jóvenes de países occidentales adoptan un estilo de vida inadecuado para su salud (12).
En Colombia (13), el $75 \%$ del sobrepeso de los estudiantes universitarios era por la baja de actividad física. Asimismo, al valorar la relación de estilos de vida con los factores cardiovasculares, las mujeres mostraron más riesgo cardiovascular, y con respecto a estilos de vida, el $46.7 \%$ de los estudiantes consumen alcohol, $9.28 \%$ tabaco y el $41.8 \%$ no realizan actividad física.

En Ecuador (10), se encontró que las mujeres tenían el perímetro abdominal aumentado (38.84\%) que en varones (19.73\%), por causa del sedentarismo, los cuales se asocian a los riesgos cardiovasculares (7), siendo el diámetro de cintura una medida principal para el diagnóstico de riesgo cardiovascular (14).

Asimismo, en Perú, (11) reportaron $12 \%$ de riesgo cardiovascular, con mayor riesgo en el género masculino $(12.1 \%)$, los cuales presentaron asociación significativamente relevante con la circunferencia de cintura.

\section{MATERIALES Y MÉTODOS}

El presente proyecto se realizó en la Universidad Peruana Unión, ubicado en la Carretera Central Km 19.5 Ñaña, Distrito de San Juan de Lurigancho, Lima. En la Facultad de Ciencias de la Salud.

\section{Muestra}

El tipo de muestreo pertenece a un estudio no probabilístico simple los participantes fueron seleccionados de acuerdo al criterio de inclusión y exclusión. El número de participantes fue de 183 estudiantes.

\section{Tipo de estudio}

El estudio es de enfoque: cuantitativo, porque mide y recoge información de los componentes del riesgo cardiovascular y del estilo de vida, utiliza instrumentos previamente existentes y validados, utiliza estadísticas, establece hipótesis y las prueba, y hace análisis de causa-efecto (15). De tipo correlacional, porque busca analizar el grado de relación entre las variables de estudio y cómo se comportan entre sí (16). Descriptivo, porque busca especificar propiedades, características y rasgos importantes (16). Además, fue de corte 
trasversal, porque los datos fueron tomados en un momento determinado (17).

\section{Instrumentos}

Se consideró como referencia inicial el instrumento llamado "Estilos de vida saludable" Originalmente creado por Arrivillaga, Salazar y Gómez (2002), presentando un análisis psicométrico según Alfa de Cronbach de 0.85 , siendo el mismo posteriormente considerado para su adaptación Palomares (2014) en la tesis de maestría titulado "Estilos de vida saludables y su relación con el estado nutricional en profesionales de la salud, en Lima - Perú" el cual presenta 48 preguntas, y está dividido en 6 componentes tales como: Condición de Actividad Física y Deporte (4 preguntas), Recreación y Manejo del Tiempo Libre (6 preguntas), Consumo de Alcohol, Tabaco y otras Drogas (6 preguntas), Sueño (6 preguntas), Hábitos Alimenticios (18 preguntas), Autocuidado y Cuidado Médico (8 preguntas), dichas preguntas fueron validados por un juicio de expertos, conformados por 3 personas.

La ficha que se utilizó para la recolección de datos antropométricos consiste en tres ítems que son: circunferencia de cintura, circunferencia de cadera e índice de cintura/cadera.

Para el inicio del proyecto de investigación se realizó los trámites de autorización con la administración de la Facultad de Salud y las escuelas de Nutrición Humana, Enfermería, Psicología y Medicina Humana, con la finalidad de obtener los permisos y presentar los objetivos del proyecto a la población seleccionado. La información se obtuvo a través de una encuesta y la medición antropométrica correspondiente.

Los instrumentos fueron aplicados durante dos semanas, el recojo de los datos duró aproximadamente 15 minutos y se contó con la ayuda de 6 profesionales. La visita se hizo individualmente por salones según los horarios accesibles, previamente se coordinó con los docentes para evitar la interrupción de las clases respectivas.

Previo a la recolección de datos se presentó el consentimiento informado (Anexo1), el cual fue leído y firmado por los estudiantes, y sirvió como autorización para la recolección de datos y participar del proyecto de investigación.
Para obtener el valor de la relación cintura cadera, se realizó con una cinta métrica conocida como Tape Measure Calculator BMI flexible e inextensible, comienza a partir de $5 \mathrm{~cm}$ y el rango de medida es de $150 \mathrm{~cm}$. El material utilizado es plástico con tamaño aprox. 7.3 $x 2.2 \mathrm{~cm} / 2.87 \times 0.87$. Es necesario realizar esta medición sin ropa o con una ropa muy ligera. Esta medición se tomó después de la aspiración normal, con los brazos relajados a cada lado; dos horas antes o después de haber ingerido alimentos.

En el momento de recoger los datos de la circunferencia cintura/cadera se colocó un ambiente adecuado para cuidar la privacidad del estudiante.

En primer lugar, se tomó la circunferencia de cintura, una medida horizontal por encima del ombligo, punto medio entre el borde inferior de la última costilla palpable y la parte superior de la cresta ilíaca, según la OMS. En segundo lugar, se toma la circunferencia de cadera, este perímetro es tomado al nivel del máximo relieve de los músculos glúteos. Estos valores deben ser apuntados y con la fórmula respectiva, calcular la relación cintura/cadera y la clase de riesgo que la persona presenta.

\section{Análisis de datos}

Para el procesamiento y análisis de datos, al obtener los datos recolectados se ingresó en una base de datos utilizando Microsoft Excel versión 2013; se utilizó también para el análisis de datos un software estadístico SSPS 24. Luego de transferir a la base de datos del software para el respectivo análisis de cada variable de estudio, se usarán pruebas de hipótesis estadísticas de acuerdo a los objetivos de investigación establecidos, tablas de frecuencias, datos porcentuales y la correlación usada será la Correlaci dee Spearm. 


\section{RESULTADOS}

En la Tabla 1 se evidencia que $67.3 \%$ de los estudiantes son de género femenino donde el $27,9 \%$ pertenecen a la escuela de Psicología y el $100 \%$ son solteros. El $85.2 \%$ de los participantes comprenden una dispersión de edad entre 18 a 23 años encontrándose un 35,0\% en la escuela de Psicología; y $49.2 \%$ son de la costa. Respecto a la condición laboral, el $77.6 \%$ son dependientes en las escuelas de Psicología y Medicina Humana encontrándose un $26,2 \%$ y $25,1 \%$ respectivamente. Finalmente, el $40.4 \%$ estudian Psicología y el $50.3 \%$ son de cuarto año.

Tabla 1

Distribución de las variables sociodemográficos de los estudiantes de la Facultad de Ciencias de la Salud.

\begin{tabular}{|c|c|c|c|c|c|c|}
\hline & Carrera & $\begin{array}{l}\text { Nutrición } \\
\text { Humana }\end{array}$ & Enfermería & Psicología & $\begin{array}{l}\text { Medicina } \\
\text { Humana }\end{array}$ & Total \\
\hline \multicolumn{7}{|l|}{ Género } \\
\hline \multirow[t]{2}{*}{ Masculino } & $\mathrm{n}$ & 9 & 2 & 23 & 25 & 59 \\
\hline & $\%$ & $4,90 \%$ & $1,10 \%$ & $12,60 \%$ & $13,70 \%$ & $32,20 \%$ \\
\hline \multirow[t]{2}{*}{ Femenino } & $\mathrm{n}$ & 22 & 22 & 51 & 29 & 124 \\
\hline & $\%$ & $12,00 \%$ & $12,00 \%$ & $27,90 \%$ & $15,80 \%$ & $67,80 \%$ \\
\hline \multicolumn{7}{|l|}{ Edad } \\
\hline \multirow[t]{2}{*}{18 a 23} & $\mathrm{n}$ & 24 & 21 & 64 & 47 & 156 \\
\hline & $\%$ & $13,10 \%$ & $11,50 \%$ & $35,00 \%$ & $25,70 \%$ & $85,20 \%$ \\
\hline \multirow[t]{2}{*}{24 a 29} & $\mathrm{n}$ & 7 & 3 & 10 & 7 & 27 \\
\hline & $\%$ & $3,80 \%$ & $1,60 \%$ & $5,50 \%$ & $3,80 \%$ & $14,80 \%$ \\
\hline \multicolumn{7}{|l|}{ Procedencia } \\
\hline \multirow[t]{2}{*}{ Costa } & $\mathrm{n}$ & 13 & 13 & 42 & 22 & 90 \\
\hline & $\%$ & $7,10 \%$ & $7,10 \%$ & $23,00 \%$ & $12,00 \%$ & $49,20 \%$ \\
\hline \multirow[t]{2}{*}{ Sierra } & $\mathrm{n}$ & 10 & 5 & 23 & 20 & 58 \\
\hline & $\%$ & $5,50 \%$ & $2,70 \%$ & $12,60 \%$ & $10,90 \%$ & $31,70 \%$ \\
\hline \multirow[t]{2}{*}{ Selva } & $\mathrm{n}$ & 7 & 3 & 6 & 4 & 20 \\
\hline & $\%$ & $3,80 \%$ & $1,60 \%$ & $3,30 \%$ & $2,20 \%$ & $10,90 \%$ \\
\hline \multirow[t]{2}{*}{ Extranjero } & $\mathrm{n}$ & 1 & 3 & 3 & 8 & 15 \\
\hline & $\%$ & $0,50 \%$ & $1,60 \%$ & $1,60 \%$ & $4,40 \%$ & $8,20 \%$ \\
\hline \multicolumn{7}{|l|}{$\begin{array}{l}\text { Condición } \\
\text { laboral }\end{array}$} \\
\hline \multirow[t]{2}{*}{ Dependiente } & $\mathrm{n}$ & 27 & 21 & 48 & 46 & 142 \\
\hline & $\%$ & $14,80 \%$ & $11,50 \%$ & $26,20 \%$ & $25,10 \%$ & $77,60 \%$ \\
\hline \multirow[t]{2}{*}{ Auto sostén } & $\mathrm{n}$ & 4 & 3 & 26 & 8 & 41 \\
\hline & $\%$ & $2,20 \%$ & $1,60 \%$ & $14,20 \%$ & $4,40 \%$ & $22,40 \%$ \\
\hline \multicolumn{7}{|l|}{ Año } \\
\hline \multirow[t]{2}{*}{ Tercero } & $\mathrm{n}$ & 16 & 12 & 36 & 27 & 91 \\
\hline & $\%$ & $8,70 \%$ & $6,60 \%$ & $19,70 \%$ & $14,80 \%$ & $49,70 \%$ \\
\hline \multirow[t]{2}{*}{ Cuarto } & $\mathrm{n}$ & 15 & 12 & 38 & 27 & 92 \\
\hline & $\%$ & $8,20 \%$ & $6,60 \%$ & $20,80 \%$ & $14,80 \%$ & $50,30 \%$ \\
\hline \multirow[t]{2}{*}{ Total } & $\mathrm{n}$ & 31 & 24 & 74 & 54 & 183 \\
\hline & $\%$ & $16,90 \%$ & $13,10 \%$ & $40,40 \%$ & $29,50 \%$ & $100,00 \%$ \\
\hline
\end{tabular}


Riesgo cardiovascular y su relación con el estilo de vida en los estudiantes de la Facultad Ciencias de la Salud de una Universidad Privada Lima, 2017

Tabla 2

Análisis de relación entre el riesgo cardiovascular y los estilos de vida saludables en estudiantes de la Facultad de Ciencias de la Salud.

$\begin{array}{llll} & & & \text { Estilo de vida } \\ \text { Rho de Spearman } & \text { Riesgo cardiovascular } & p & 0.234 \\ & n & 183\end{array}$

Tabla 3

Análisis de relación entre el riesgo cardiovascular y los estilos de vida en estudiantes según las dimensiones.

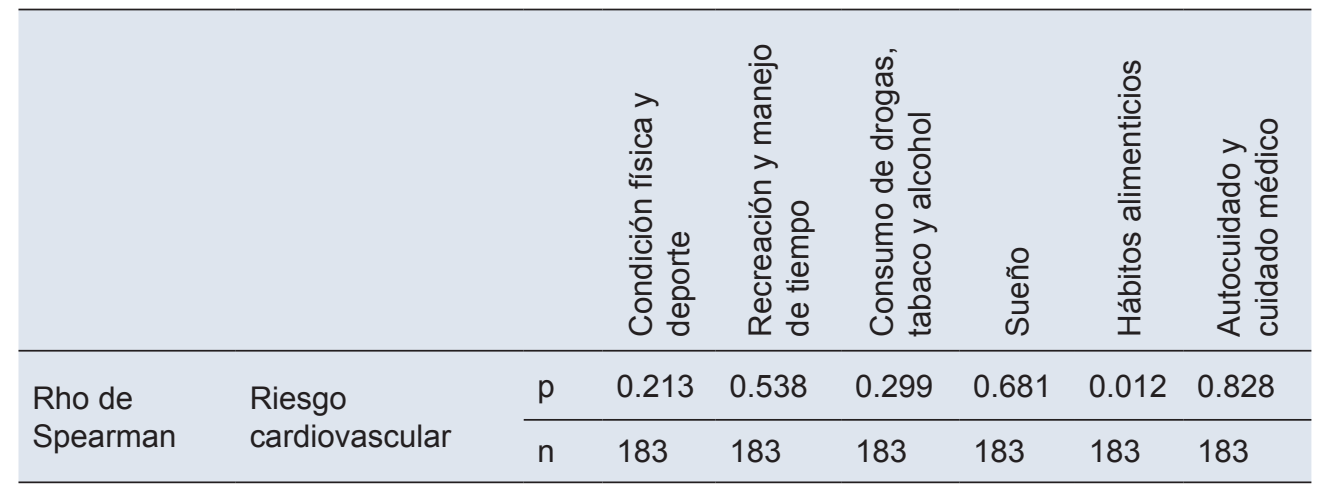

\section{Gráfico 1}

Descripción del riesgo cardiovascular de los estudiantes de la Facultad de Ciencias de la Salud.

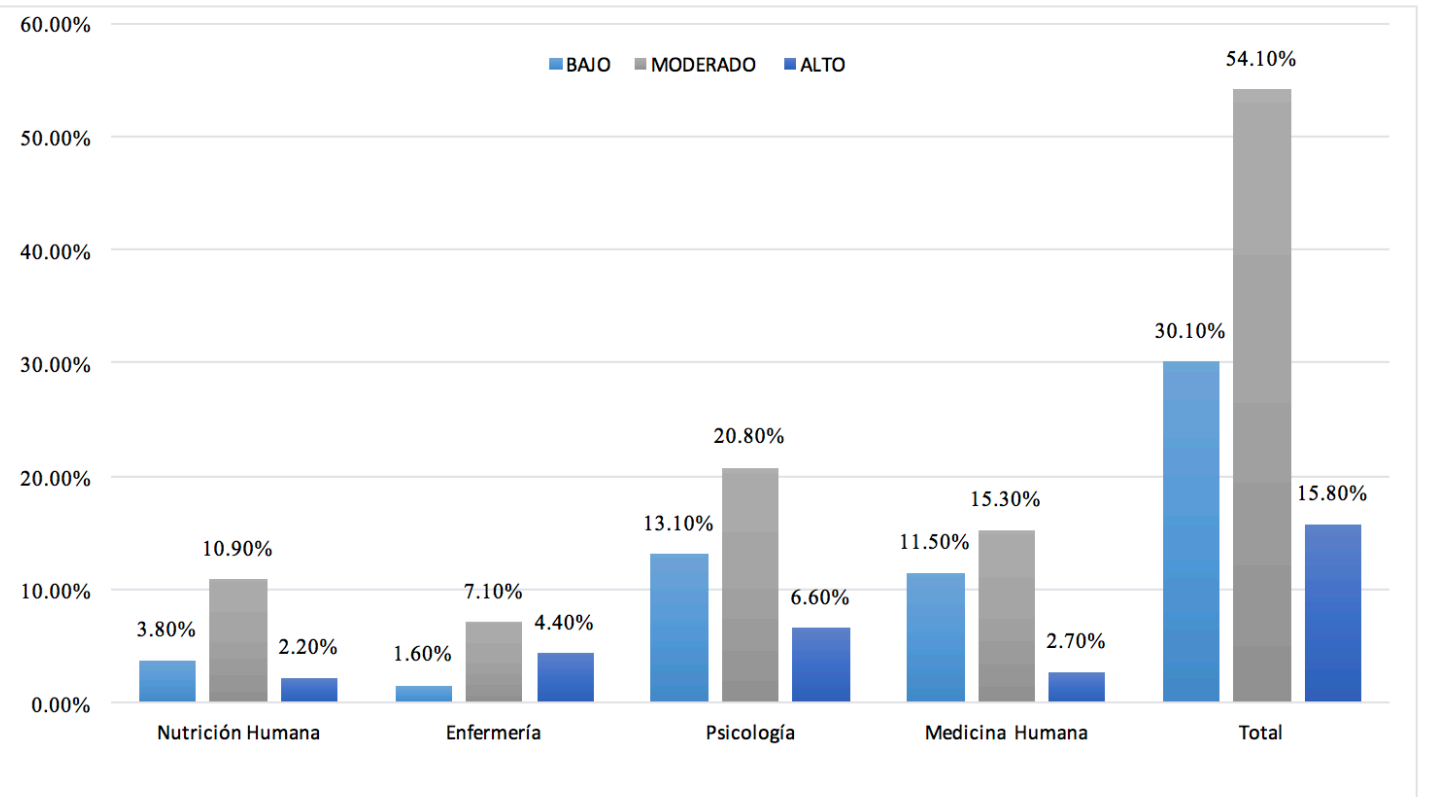


Gráfico 2

Descripción del estilo de vida saludable de los estudiantes de la Facultad de Ciencias de la Salud.

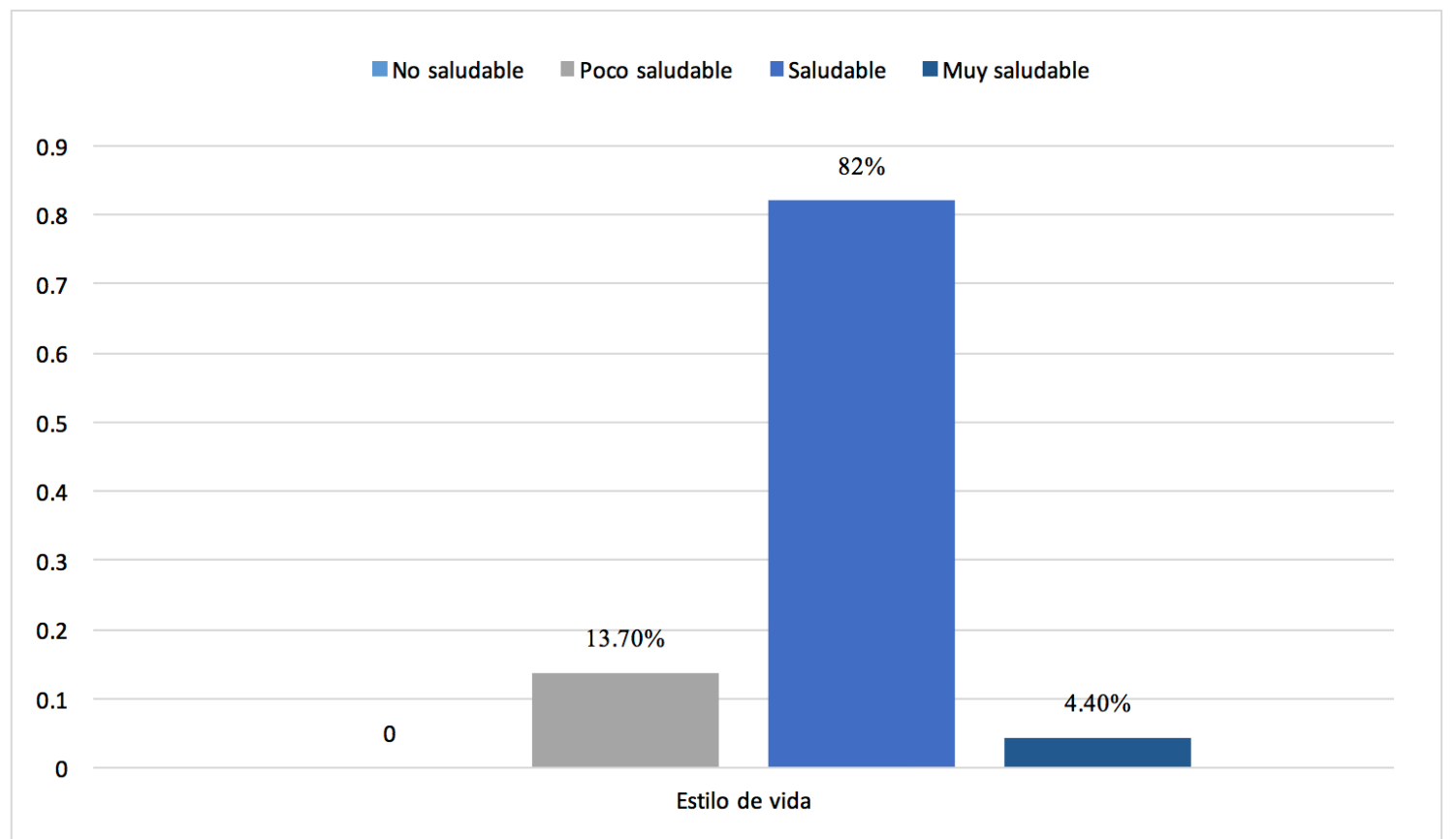

\section{DISCUSIÓN}

El riesgo cardiovascular estudiado en la población universitaria en estudiantes de la FCS reportó que el $54.10 \%$, presentó un riesgo cardiovascular moderado, (M: 0.75- 0.85; V: 0.90 - 1.00) según los limites establecido por Saavedra et al (18). En cuanto al estilo de vida, el $82 \%$ de los estudiantes son saludables, mientras que en el estudio "Evaluación de hábitos de salud e identificación de factores de riesgo en estudiantes de la División de Ciencias Naturales y Exactas", realizado en México, se encontró que un $57 \%$ presentaron malos estilos de vida, siendo representativo los hábitos alimenticios deficientes (19). Entre seis dimensiones que comprende esta variable, se observó que los estudiantes presentaron una condición física y deporte poco saludable, un $48,6 \%$. Un estudio similar realizado en México por Piña et al (19) en universitarios, observó que el $40 \%$ de la población tiene hábitos sedentarios. Adicionalmente Cruz et al (20) en un estudio transversal encontró que el $55.9 \%$ realiza actividad física leve, mientras que Padilla et al (13) encontró que el $41.8 \%$ de los universitarios no realiza ningún tipo de actividad física. La segunda dimensión estudiada es la recreación y manejo del tiempo libre, se encontró que el $50.8 \%$ de los estudiantes era saludable; un estudio similar realizado por Morales (21) en universitarios de Lima respalda estos resultados, siendo que más de la mitad $(59,0 \%)$ de la población estudiada alcanzaron un nivel alto de actividad física y menos horas en sus momentos de ocio. La tercera dimensión es el consumo de drogas, alcohol y tabaco, el $85,8 \%$ de los estudiantes se presentaron muy saludables, en contraste con el estudio de Almonacid et al (22) en Colombia donde el consumo de alcohol fue de $96.1 \%$ y tabaquismo de $48.1 \%$; asimismo en Chile, Morales et al (23) encontró un consumo de alcohol de $75,5 \%$. Asimismo para la cuarta dimensión, de sueño se encontró un $66,1 \%$, es decir que los participantes son saludables, a diferencia del estudio realizado por Piña et al (19) en universitarios de México donde el $66 \%$ no duermen las horas adecuadas. Los hábitos alimentarios, quinta dimensión del presente estudio, se encontró que el $50,3 \%$ se mantienen saludables; asimismo, al comparar con otra población estudiantil de la Universidad de Colombia se presentaron saludables con un resultado del $56,1 \%$, este fue un estudio realizado por Bennassar (24), Muñoz et al (25) evaluaron la dieta de los estudiantes y se encontró que $80.2 \%$ estuvieron en la categoría "poco saludable", 19,7\% en "necesita cambios" y tan solo $0,1 \%$ en "saludable". La última dimensión de la variable estilos de vida es el 
autocuidado y cuidado médico encontrándose en este estudio que, $55,6 \%$ son poco saludables en cuanto al autocuidado cuidado médico, Escobar y Pico (26) reportaron en su estudio que el $57,2 \%$ de los estudiantes no dispone del "tiempo para ir a las citas con profesionales de la salud y para dedicarle al cuidado personal.

Se conoce que durante el transcurso de los ciclos académicos, los cambios de comportamiento son notorios en los universitarios, debido a la presión académica y el estrés afecta directamente la salud de los estudiantes, Núñez et al (27) menciona que en los grupos de mayor edad, es decir universitarios se ve afectada su composición corporal, la obesidad y sobrepeso especialmente, y en particular la obesidad abdominal, Zea et al (4) respalda esta afirmación, refiere que los hábitos que llevan los estudiantes universitarios tiene relación directa en la prevalencia de problemas cardiovasculares e incluso la composición corporal; por tanto se sugiere modificaciones en cada aspecto al que se refiere el término estilo de vida. La intervención de programas bien planteados tiene buenas referencias sobre la reducción de los factores de riesgo a través de programas en estilos de vida, Leiva et al (28) aplicaron un programa donde la actividad física (29) y hábitos alimentarios fueron los ejes de trabajo; los resultados fueron positivos y resultaron ser efectivos en la reducción de los FRCV.

En cuanto a la relación entre riesgo cardiovascular y estilos de vida, los resultados del presente estudio mostraron un valor $p>.05 \quad(p=0.234)$, por tanto, no existe relación estadísticamente significativa entre riesgo cardiovascular y estilos de vida. De igual manera Ñuñuvera (30) en Trujillo, Perú concluyó que no existe evidencia suficiente para afirmar que existe relación entre los factores de riesgo cardiovasculares: consumo de alcohol $(p=0,30)$, sedentarismo $(p=0,069)$, obesidad $(p=0,65)$ y consumo de tabaco $(p=0,328)$, y los estilos de vida. De igual manera Gómez et al (31) identificaron riesgo cardiovascular en $40 \%$ de los hombres y $35 \%$ de las mujeres al presentar circunferencia de cintura $>90$ y $>80 \mathrm{~cm}$ respectivamente, sin embargo al considerar el ICC, se encontró un riesgo bajo en ambos sexos, presentaron mayor prevalencia de sobrepeso y obesidad con diferencia significativa $(p<0.028)$. Sin embargo Reguera et al (32) en México en su estudio titulado "Estilo de vida y factores de riesgo en estudiantes de la Universidad de Sonora" encontraron tres factores de riesgo en los universitarios: sedentarismo $(p<0.000)$, consumo abusivo del alcohol $(p=0.01)$ y conductas sexuales de riesgo $(p=0.086)$. No se observó evidencia estadísticamente significativa en los hábitos de alimentación $(p=0.750)$. Cabe mencionar que en España, un estudio realizado por Rodríguez (33), consiguió cambios favorables al aplicar el programa de cambio de estilo de vida en parámetros como el peso $(p<0.01)$, la cintura $(p<0.001)$, el $\%$ de masa grasa $(p<0.05)$ y masa magra $(p<0.05)$.

Analizando la relación entre la variable riesgo cardiovascular y las dimensiones de estilos de vida, se obtuvo lo siguiente: El riesgo cardiovascular y la condición física y deporte no existe relación estadísticamente significativa $p>.05(p=.2130)$, Moreno (34) encontró en estudiantes de Colombia que los estudiantes de mayor edad (20-27 años) presentan mayor riesgo de sedentarismo que en jóvenes (17- 19 años) $(p=0,009)$, encontró que los estudiantes sedentarios tienen una frecuencia cardiaca mayor comparada con los estudiantes no sedentarios $(p=0,040)$, lo que representa que el riesgo cardiovascular es mayor en aquellos que no cuidan de su condición física y deporte, sin embargo en cuanto al ICC no se encontró evidencia suficiente para demostrar la relación entre el riesgo cardiovascular y sedentarismo $(p=0,708)$. De igual manera en Chile, Alarcón et al. (5) en su estudio estado nutricional, niveles de actividad física y factores de riesgo cardiovascular en estudiantes de la Universidad Santo Tomás evidenciaron que no existe relación entre factores de riesgo cardiovascular y el contorno de cintura $(p=0,202)$ y tampoco encontraron relación entre actividad física y contorno cintura $(p=0.919)$. Sin embargo en el mismo estudio encontraron diferencias significativa en el colesterol HDL se mostró disminuido en los estudiantes activos $(p=0,016)$ y el colesterol total en los sedentarios $(p=0,027)$ se observó levemente aumentado. En Chile, Caamaño et al (12) en su estudio niveles de obesidad, perfil metabólico, consumo de tabaco y presión arterial en jóvenes sedentarios reporta una elevada prevalencia de exceso de peso y sedentarismo, mostrando que existe una relación entre contorno cintura y la obesidad $(p=$ $0,000)$; Valcárcel (35) concluye que la práctica de ejercicio aeróbico regular puede inhibir la progresión de lesiones coronarias y, en algunos casos, se observa regresión de la enfermedad en pacientes que han modificado sus FRC 
y practican ejercicio aeróbico regularmente. Esto se respalda con Hernández (36) en donde menciona que la reducción del riesgo de muerte fue de $29 \%$ al $67 \%$ en los pacientes que realizaban altos niveles de actividad física, y del $12 \%$ al $35 \%$ en los pacientes que realizaban niveles moderados. Sin embargo en un estudio realizado en Lima, Quispe et al (21) muestra un nivel alto de actividad física en dicha población, los resultados que obtuvieron fueron mayores niveles de actividad física en las áreas "labores de hogar" y "recreación". La actividad física en las labores del hogar fue mayor en el sexo femenino $(p=0,010)$, mientras que en las actividades de recreación la AF fue mayor en los varones $(p=0,009)$.

En la relación de riesgo cardiovascular y recreación no existe relación estadísticamente significativa, siendo $p>.05 \quad(p=0.538)$. La educación de los estudiantes inician en el hogar, la familia debe ser consciente del impacto que genera el estilo de vida en su salud, incentivar al manejo correcto del tiempo libre. Santaliestra (37) menciona que durante los últimos años $1 / 3$ de los niños no cumplen las recomendaciones del tiempo frente a una pantalla, los adolescentes y jóvenes exceden el tiempo en internet y en jugar a los ordenadores, y como resultado existe un mayor consumo de refrescos azucarados y menor consumo de fruta. Estos patrones dietéticos con el tiempo invertido son parte de las conductas sedentarias. En los jóvenes un mayor consumo de tiempo frente a un monitor o pantalla de celular y utilizando internet en su tiempo libre está relacionado con menor adherencia a los patrones saludables, estas conductas se consideran obesogénicas y por lo tanto son un factor de riesgo cardiovascular. En un estudio realizado por Campo et al (38) en España identificó que los principales motivos de los estudiantes para no realizar actividades de ocio son: no saber cómo ocupar el tiempo libre o preferir. Así también los estudiantes universitarios dedican una media de 1,7 horas al día a ver la televisión en su tiempo libre (1,5 horas entre semana y 2,0 horas durante el fin de semana), refieren que los varones son los que tienen una mayor dedicación a los videojuegos. Un estudio realizado en Lima Perú, por Quispe et al (21) menciona que los adolescentes que tienen acceso a Internet con una frecuencia de 2 a 3 veces/semana son los que en mayor proporción obtienen nivel alto de actividad física. Según el uso de internet en horas por semana, la mayoría consumen de 3 a $5 \mathrm{~h}$ por semana $y$, de este grupo, la mayor proporción obtiene nivel de actividad física alto; entre los que usan más de $20 \mathrm{~h}$ de Internet por semana, el 58,3\% obtiene nivel de actividad física moderado. Sin embargo no se encontró asociación entre el nivel de actividad física y el sexo, grupo de edad, tiempo de residencia, frecuencia de acceso a Internet ni consumo en horas por semana $(p>0,05)$. Mientas que en el estudio hecho en Caracas (Venezuela) por Navas (39) refiere que los estudiantes si dan valor e importancia a la Recreación y consideran que contribuye de una manera significativa en su formación integral, la preferencia estuvo centrada en actividades recreativas del área de vida al aire libre y turismo reflejando porcentajes de aceptación sobre el $80 \%$ y de $85 \%$ respectivamente quedando en segundo lugar el área socio-cultural y deporte pues en todo momento mostraron una actitud positiva y abierta frente a la importancia de la recreación y el tiempo libre como alternativa para mejorar su calidad de vida y su aprendizaje profesional.

En cuanto a la relación de riesgo cardiovascular y consumo de drogas, tabaco y alcohol en el presente estudio no se encontró relación estadísticamente significativa $p>.05 \quad(p=0.299)$. El motivo por la que no se encontró relación estadísticamente significativa entre ambas variables, se debe al tipo de población en donde se realizó el estudio, la filosofía de una universidad adventista se basa en que el estilo de vida saludable se enfoca a la dieta saludable, consumo de agua, respiración de aire puro, actividad física y descanso adecuado, excluyendo el consumo de alcohol, café, tabaco y drogas psicoactivas (40). Sin embargo en el estudio hecho por Almonacid et al (22) titulado evaluación de factores de riesgo asociados a enfermedad cardiovascular en jóvenes universitarios, el estilo de vida no se basa en la filosofía mencionada anteriormente al referirnos específicamente en el hábito de fumar, se encontró mayor la frecuencia en los hombres que en las mujeres $(P<0.001)$. También demostraron que las mujeres tienen ICC más altos que los hombres $(P<0.05)(p=.030)$, y a raíz del estudio se encontró una asociación estadísticamente significativa de la presencia de presión arterial media-alta con el hábito de fumar $(P \leq 0,001)$ y el consumo de alcohol una vez a la semana $(P=0.001)$. En el aspecto metabólico $65.48 \%$ de las mujeres con sobrepeso tuvo niveles bajos de cHDL $(P=0.029)$, por lo tanto hubo asociación estadísticamente significativa entre 
la obesidad y los altos niveles de triglicéridos ( $\mathrm{P}$ $=0.001$ ). Reguera et al (32) en el estudio estilo de vida y factores de riesgo en estudiantes de la Universidad de Sonora, aunque no se encontró la relación entre el ICC y el hábito de consumir drogas, alcohol y drogas se encontró una relación significativa entre los estilos de vida y consumo de tabaco y alcohol $(p=0.01)$, lo cual es uno de los factores de riesgo cardiovascular. Salazar y Arrivillaga (41) en Colombia en su estudio titulado: El consumo de alcohol, tabaco y otras drogas, como parte del estilo de vida de jóvenes universitarios, encontró que el $82 \%$ fuma en la casa $(p=0.002)$, el $89 \%$ lo hace en la universidad ( $p=0.000$ ), y el $73 \%$ fuma en casa de amigos $(p=0.001)$. Igualmente, el $77 \%$ acepta que consume licor con sus amigos $(p=0.000)$ y el $78 \%$ dice que en su vida social habitualmente se consume licor $(p=0.000)$.

En la relación entre el riesgo cardiovascular y el sueño en estudiantes universitarios se encontró un valor de $p>.05(p=0.681)$ es decir no existe relación estado estadísticamente significativa, sin embargo Durán et al (42) con el título menos horas de sueño asociado con sobrepeso y obesidad en estudiantes de nutrición de una universidad chilena, refiere que el $57,1 \%$ de universitarios duermen menos de lo recomendado, concluyen que existe asociación entre menos horas de sueño y un mayor peso corporal de los estudiantes $p>.05$ $(p=0,020)$. En Chile, Valladares et al (43) en su estudio titulado "Asociación entre cronotipo y obesidad en jóvenes", presentó que los hombres con cronotipo trasnochador presentaron significativamente mayor perímetro de cintura ( $p$ $=0,03$ ). Las mujeres con un porcentaje de grasa $<25 \%$ se asoció con cronotipo trasnochador ( $p$ $=0,05)$. En relación al cronotipo y porcentaje de grasa corporal se observó en aquellas mujeres que tienen un porcentaje de grasa corporal mayor al $25 \%$ (catalogadas como obesas) tienen valores significativamente más bajos en los puntajes de cronotipo ( $p=0.05)$, lo que se asocia a un fenotipo trasnochador. Monge (44) relaciona las escasas horas de sueño como factor colaborador en la génesis de la obesidad. Núñez et al (27) hizo un estudio: titulado prevalencia de factores de riesgo cardiovascular y riesgo metabólico en escolares, universitarios y mujeres de organizaciones sociales de base en distritos de Lima, Callao, La Libertad y Arequipa, Perú; en donde los universitarios presentaron un porcentaje de obesidad abdominal del $35,0 \%$, también el $47,7 \%$ de los estudiantes procedentes de Chaclacayo Lima tenían una inadecuada cantidad de sueño. Se encuentra que el envejecimiento precoz en individuos obesos y crónicamente privados del sueño eleva el riesgo cardiovascular, esto lo confirma Miller et al (45) que las horas de acostarse tarde se asociaron con la adiposidad, independientemente de la duración del sueño. Finalmente Ramón et al (46) menciona que las calificaciones más altas académicamente son el resultado de patrones de descanso adecuados y el ejercicio moderado después ( 2 a 5 horas a la semana).

La relación entre riesgo cardiovascular y hábitos alimenticios del estudio tuvo una relación estadísticamente significativa $p<.05$ $(p=0.012)$, es decir que existe una relación alta entre el riesgo cardiovascular y hábitos alimenticios. En España, Bennassar et al (24) en su estudio "Estilos de vida y salud en estudiantes universitarios" encontró que los estudiantes presentan baja adherencia a los hábitos alimenticios saludables y actividad física. Cabe destacar la correlación entre el estado de salud y la calidad de vida $(p<0,01)$. En el momento de realizar el estudio el $78,4 \%$ de los estudiantes universitarios no realizaba ninguna dieta o régimen especial frente al $21,6 \%$ que sí. De estos últimos, el 1,2\% realizaba una dieta especial para ganar peso, el 17,6\% para perderlo y el 2,8\% por algún problema de salud. Se observan diferencias significativas entre la realización de alguna dieta o régimen especial y el sexo, de forma que el doble de mujeres $(22,1 \%)$ que de hombres $(10,5 \%)$ realizan una dieta $o$ régimen para perder peso $(p=0,006)$. Con estas evidencias podemos reafirmar que dicha población por lo general no cumple hábitos alimenticios saludables, siendo que la mayoría consume dietas desequilibradas con alto aporte calórico lo cual es un factor determinando para el riesgo cardiovascular. También Rodriguez (33) en su estudio encontró que las mujeres parecen estar más preocupadas por mantener una alimentación saludable, teniendo más cuidado con los alimentos que eligen y la forma de cocinarlos (tomar verdura y alimentos frescos, no abusar de los fritos y de las grasas, etc.) $(p<0.05)$.

En 2013, en España (47) se aplicó un método de muestreo en dos etapas. La primera etapa, las unidades que se muestreaban fueron ciudades y entidades locales y, en la segunda, se seleccionaron los hogares que conformaron la muestra final a partir de las entidades locales. 
A través de una encuesta de frecuencia de consumo, encontró que el mayor aporte de energía procedía de los lípidos, también se identificó que el sodio se asocia a un mayor desarrollo de la aterosclerosis (48) porque que actúa a través de la activación del sistema renina-angiotensina-aldosterona, lo que lleva al desarrollo de las enfermedades cardiovasculares como lo afirma Vivanco et al (49).

Finalmente el presente estudio concluye al relacionar el riesgo cardiovascular y autocuidado y cuidado médico, no existió relación estadísticamente significativa con un valor $p$ $>.05$ ( $p=0.82$ ); así mismo, en universitarios colombianos de la Facultad de Ciencias de la Salud realizado por Escobar y Pico (26) también encontraron que las prácticas de autocuidado de la salud fueron saludables $(p=0,000)$ al igual que en el presente estudio, llamó la atención que los varones dan menos importancia a la realización del autoexamen físico, lo cual es estadísticamente significativo $(p=0,000)$, y las mujeres se presentan más prácticas de autocuidado y en cuanto a la exploración del cuerpo para detectar cambios, los hombres son los que menos la realizan $(p=0,116)$. Al comparar las prácticas de cuidado entre quienes se ubican en cada etapa de cambio, se encuentran diferencias estadísticamente significativas $(p=$ 0,000 ), siendo más saludables las prácticas en quienes consideraron "sentirse satisfechos y no piensan que sean problemáticas". Así mismo Gómez (50) estudió a 570 adultos-jóvenes en Bogotá (Colombia) reportó que las creencias saludables y el autocuidado en salud, predicen a los estilos de vida saludables en un $39 \%$, se registró una correlación positiva $(r=0,23)$ con el autocuidado médico y la prevención de riesgos en salud. Sin embargo Morales y Flores (51) en su estudio titulado agencia de autocuidado y factores de riesgo cardiovascular, aplicaron la Escala Apreciación de la Agencia de Autocuidado a 133 estudiantes al relacionar el autocuidado y el riesgo cardiovasculares encontraron un valor mayor a $p<. .05$ demostrando las probabilidades de desarrollar alguna enfermedad cardiovascular en el futuro, al analizar la variable perímetro abdominal, con base en la clasificación del perímetro abdominal, se observó que el $61 \%$ de adolescentes hombres y el $39 \%$ de adolescentes mujeres no mostró riesgo para obesidad. Sin embargo, el $80 \%$ de las mujeres y el $20 \%$ de los hombres se encontraban en estado de alerta.

\section{CONCLUSIÓN}

- En cuanto al estilo de vida, el $82 \%$ de los estudiantes son saludables.

- El riesgo cardiovascular estudiado en la población universitaria de la FCS reportó que el $54.10 \%$, presentó un riesgo cardiovascular moderado, según las dimensiones del estilo de vida se encontró que:

- Los estudiantes presentaron una condición física y deporte poco saludable, es decir un $48,6 \%$.

- En recreación y manejo del tiempo libre, se encontró que el $50.8 \%$ de los estudiantes era saludable.

- El consumo de drogas, alcohol y tabaco, representa un $85,8 \%$ por lo tanto son muy saludables.

- En horas de sueño, se encontró un 66,1\%, mostrando que los participantes son saludables.

- En los hábitos alimentarios, se encontró que el $50,3 \%$ se mantienen saludables.

- En autocuidado y cuidado médico son el $55,6 \%$, es decir son poco saludables.

- En la relación de estilos de vida y riesgo cardiovascular, solamente se encontró relación en la dimensión hábitos alimenticios.

\section{RECOMENDACIONES}

Para estudios posteriores se recomienda, ampliar la muestra, considerándose todos los ciclos académicos del primero al décimo, también considerar un estudio longitudinal para recolectar la data en tres momentos, al inicio, medio y final del ciclo de estudio, asimismo considerar otras variables como valores de perfil lípidos entre otros, siendo que es un factor que sumaría a un riesgo cardiovascular elevado.

También fomentar la práctica constante de un estilo de vida saludable como parte fundamental de la formación académica, a través de una planificación de diversas actividades que involucre docentes y/o estudiantes para generar 
una adherencia a buenos hábitos alimenticios, de actividad física y deporte, control de estrés, autocuidado médico entre otros componentes de suma importancia, para tener una formación integral como profesionales de la salud que brinden un servicio con testimonio.

\section{Declaración de financiamiento y de conflictos de interés:}

El estudio fue financiado por los autores, quienes declaran no tener conflictos de interés.

\section{Correspondencia}

Tania Zhunaula Guamán

Correo electrónico: pool_tania93@hotmail.com

\section{REFERENCIAS BIBLIOGRÁFICAS}

Organizacion Mundial de la Salud (OMS). Centro de prensa Enfermedades cardiovasculares. Enfermedades Cardiovasc. 2015;1-6.

Celada P, Delgado G, Olmedilla B, Jiménez F, Ruperto M, Sánchez F. Impact of improved fatmeat products consumption on anthropometric markers and nutrient intakes of male volunteers at increased cardiovascular risk. Nutr Hosp. 2015;32(2):710-21.

Cedeño S, Goicoechea M, Torres E, Verdalles Ú, José $A$, Verde $E$, et al. Predicción del riesgo cardiovascular en pacientes con enfermedad renal crónica. Rev la Soc Española Nefrol. 2017;7(3):293-300.

Zea $A$, León $H$, Botero $D$, Afanador $H$, Pinzón L. Factores de riesgo cardiovascular y su relación con la composición corporal en estudiantes universitarios. Rev Salud Pública. 2014;16(4):505-15.

Alarcón M, Delgado P, Caamaño F, Osorio A, Rosas M, Cea F. Estado nutricional, niveles de actividad física y factores de riesgo cardiovascular en estudiantes de la Universidad Santo Tomás. Rev Chil Nutr. 2015;42(1):70-6.

Organizacion Mundial de la Salud. Centro de prensa: Actividad física. OMS. 2017;1-5.

Delgado $\mathrm{P}$, Alarcón M, Caamaño F. Análisis de los factores de riesgo cardiovascular en jóvenes universitarios según su estado nutricional. Nutr Hosp. 2015;32(4):1820-4.
Caamaño F, Delgado P, Guzmán I, Jerez D, Campos C, Osorio A. La malnutrición por exceso en niños-adolescentes y su impacto en el desarrollo de riesgo cardiometabólico y bajos niveles de rendimiento físico. Nutr Hosp. 2015;32(6):257683.

Serrat S. Dieta, estilos de vida y factores de riesgo cardiovascular en niños y adolescentes europeos. 2014 ;

Ruano C, Melo J, Mogrovejo L, De Paula K, Espinoza C. Prevalencia de síndrome metabólico y factores de riesgo asociados en jóvenes universitarios ecuatorianos. 2015;31(4):1574-82.

Pajuelo J, Sánchez J, Álvarez D, Tarqui C, Bustamante A. La circunferencia de la cintura en adolescentes del Perú. An Fac Med. 2016;77(3).

Caamaño F, Alarcón M, Delgado P. Niveles de obesidad, perfil metabólico, consumo de tabaco y presión arterial en jóvenes sedentarios. Nutr Hosp. 2015;32(n05):2000-6.

Padilla C, Jaimes M, Fajardo S, Ramos A. Factores de riesgo cardiovascular y estilos de vida de estudiantes universitarios. 2014;17(2):81-90.

González C, Díaz Y, Mendizabal A, Medina E, Morales J. Prevalencia de obesidad y perfil lipídico alterado en jóvenes universitarios. Nutr Hosp. 2014;29(2):315-21.

Hernández R, Fernandez C, Baptista P. Metodología de la investigación. Vol. 53, Journal of Chemical Information and Modeling. México; 2010. 656 p.

Díaz V, Calzadilla A. Artículos científicos, tipos de investigación y productividad científica en las Ciencias de la Salud. Rev Cienc Salud. 2016;14(1):115-21.

Álvarez G, Delgado J. Diseño de estudios epidemiológicos. El estudio transversal: Tomando una fotografía de la salud y la enfermedad. Bol Clin Hosp Infant Edo Son. 2015;32(1):26-34.

Saavedra S, Waitman J, Cuneo C. Obesidad. Rev Fed Argentina Cardiol. 1999;(Tabla I):28-30.

Piña B, Alvarado A, Deveze M, Durán E, Padilla F, Mendoza C. Evaluación de hábitos de salud e identificación de factores de riesgo en estudiantes de la División de Ciencias Naturales y Exactas (DCNE ), unidad. 2015;25(477):68-75.

Cruz E, Osorio M, Bernardino A, Vásquez L, Galindo $\mathrm{N}$, Grajales I. Factores de riesgo cardiovascular en estudiantes de enfermería de una universidad pública. Enfermería Univ. 2016;13(4):246-52. 
Quispe J, Añez R, Suarez C. Nivel de actividad física en adolescentes de un distrito de la region Callao. Rev Peru Med Exp Salud Publica. 2016;33(2):18.

Almonacid C, Camarillo M, Gil Z, Medina C, Rebellón $\mathrm{J}$, Mendieta H. Evaluación de factores de riesgo asociados a enfermedad cardiovascular en jóvenes universitarios de la localidad Santafé en Bogotá, Colombia. 2016;35-46.

Morales G, Guillen F, Muñoz S, Belmar C, Schifferli I, Muñoz A, et al. Factores de riesgo cardiovascular en universitarios de primer y tercer año. Rev Med Chil. 2017;145(3):299-308.

Bennassar M. Estilos de vida y salud en estudiantes universitarios: La universidad como entorno promotor de la salud. Vol. 4, Saude e Sociedade. Universitat de les Illes Balears; 2010.

Muñoz J, Córdova J, Del Valle D. El índice de alimentación saludable de estudiantes de nuevo ingreso a una universidad de México. 2015;31(4):1582-9.

Escobar M, Pico M. Autocuidado de la salud en jóvenes universitarios, Manizales, 2010-2011. RevFac NacSalud Pública. 2013;2010-1.

Nuñez E, Huapaya C, Torres R, Esquivel S, Suarez V, Yasuda M, et al. Prevalencia de factores de riesgo cardiovascular y riesgo metabólico en escolares, universitarios y mujeres de organizaciones sociales de base en distritos de Lima,Callao,La libertad y Arequipa, Perú 2011. Rev Peru Med Exp Salud Publica. 2014;31(4):652-60.

Leiva A, Martínez M, Celis C. Efecto de una intervención centrada en la reducción de factores de riesgo cardiovascular en estudiantes universitarios. Rev Med Chil. 2015;143(8):971-8.

Zafra M. Resultados de la aplicacion de un programa de ejercicio fisico en la mejora de los factores de riesgo cardiovascular y condición física en adultos: Programa ACTIVA. 2015;

Ñuñuvera M. Estilos de vida y su relacion con factores de riesgo cardivoascular en adolescentes del Colegio Maria Negrón Ugarte Trujillo- Perú 2012. 2013;1(1):11-9.

Gomez Z, Laderos P, Romero E, Troyo R. Estilo de vida y riesgo para la salud en una poblacion universitaria. Rev Salud Publica y Nutr [Internet]. 2016;15(2):15-21. Available from: http://www. medigraphic.com/pdfs/revsalpubnut/spn-2016/ spn162c.pdf

Reguera M, Sotelo N, Barraza A, Cortez M. Estilo de vida y factores de riesgo en estudiantes de la
Universidad de Sonora : Un estudio exploratorio. Boletín Clínico del Hosp Infant del Estado Son [Internet]. 2015;32(2):63-8. Available from: http://eds.b.ebscohost.com/eds/pdfviewer/ pdfviewer?sid=5cbf0d80-1a42-449f-a9c45625da4c5a51@sessionmgr103\&vid=1\&hid=127

Rodríguez B. Evaluacion de una estrategia grupal y multidisciplinar de cambio de estilo de vida en obesidad. Universidad Complutense de Madrid; 2013.

Moreno J, Cruz H, Angarita A. Evaluación de razones de prevalencia para sedentarismo y factores de riesgo en un grupo de estudiantes universitarios. Chía- Colombia. Enferm Glob. 2014;13(34):11422.

Valcárcel G. Marcadores bioquímicos de riesgo cardiovascular: efecto del ejercicio físico y del entrenamiento. Universidad de Oviedo; 2015.

Hernández K, Pérez M, Arias P. La actividad física reduce el riesgo de muerte en pacientes con hipertensión. Rev Duazary. 2017;14(1):91-100.

Santaliestra A. Patrones de sedentarismo y su relación con la ingesta de alimentos en niños y adolescentes europeos. 2015;76-84.

Campo Y, Pombo L, Teherán A. Estilos de vida saludable y conductas de riesgo en estudiantes de medicina. 2016;48(3):301-9.

Navas L. Diseño de un programa de recreación organizada dirigido a los estudiantes del Instituto pedagógico de Caracas. Rev Invest (Guadalajara). 2015;39:155-72.

Pinzón D. Estilo de vida saludable (EVS): limitaciones del enfoque biomédico. Rev Apunt Univ [Internet]. 2014;3(1):9-26. Available from: http://dialnet. unirioja.es/servlet/articulo?codigo $=4331970$

Salazar I, Arrivillaga M. El consumo de alcohol, tabaco y otras drogas, como parte del estilo de vida de jóvenes universitarios. 2004;1-14.

Durán S, Fernández E, Fehrmann P, Delgado C, Quintana C, Yunge W, et al. Menos horas de sueño asociado con sobrepeso y obesidad en estudiantes de nutrición de una Universidad Chilena. Rev Peru Med Exp Salud Publica. 2016;33(2):264-8.

Valladares M, Campos B, Zapata C, Duran S, Obregón A. Asociación entre cronotipo y obesidad en jóvenes. 2016;33(6):1336-9.

Monge L. Valoración de la masa grasa mediante dxa y su relación con variables e índices antropométricos. 2015. 
Miller A, Lumeng J, Lebourgeois M. Sleep patterns and obesity in childhood. 2015;22(1):41-7.

Ramón C, Clariana M, Gotzens C, Badia M, Dezcallar T. Patrones de descanso, actividades físico deportivas extraescolares y rendimiento académico en niños y niñas de primaria. Rev Psicol del Deport. 2015;24(1):53-9.

Varela G, Ruiz E, Valero T, Ávila J, Pozo S. The spanish diet : an update. Nutr Hosp. 2013;28:1320.

Montes S, Benet M, Ramos L, Cano E, Perez E. Factores de riesgo cardiovasculares e hiperreactividad cardiovascular en jóvenes venezolanos. 2017;108-17.
Gómez A. Predictores psicológicos del autocuidado en salud. 2017;7577(1):0-2.

Vivanco G, Rodriguez J, Torres E, Martínez M, Gonzalez M. Enfermedades cardiovasculares, hipertensión arterial y consumo de sodio: una relación controversial. Rev Iberoam las Ciencias la Salud. 2016;5(10).

Morales R, Flórez M. Agencia de autocuidado y factores de riesgo cardiovascular en adolescentes. (Spanish). Self-care agency Cardiovasc risk factors Adolesc. 2016;34(2):125-36.

Recibido: $15 / 01 / 18$

Aceptado: 09/05/18 